\title{
SKINFOLD TECHNIQUES WHEN PROVIDING SUBCUTANEOUS INJECTIONS
}

\author{
Bibi Bond, Veerle Wille, Jolanda Maaskant \\ Emma Children's Hospital, Amsterdam UMC - Location AMC, Amsterdam, The Netherlands
} b.bond@amc.uva.nl

\section{Introduction}

Many children with various diseases receive medicines by subcutaneous injections as part of their treatment. It is unclear how to hold the skinfold during this procedure in order to provide the medicine correctly. This variation in care is undesirable for nurses, and for children and parents who are taught to inject subcutaneously. Therefore, we systematically searched the literature that investigated the effectiveness and safety of different skinfold techniques when providing subcutaneous injections in pediatric patients.

\section{Methods}

We researched PubMed and CINAHL for studies published between 2007 and 2017. No further limits were applied. The articles were selected on title, abstract and full-text according predefined inclusion and exclusion criteria. Tools of the Cochrane Collaboration were used for quality assessment and data extraction.

\section{Results}

We included 4 moderate quality studies.

- The overall results are congruent and show that nonpinched skinfold technique and needle length are associated with an increased risk of incorrect subcutaneous injections $(p<0.0001)$.

- Unintended intramuscular injections occurred more frequently with vertically inserted $6 \mathrm{~mm}$ and with $45^{\circ}$ angle inserted $8 \mathrm{~mm}$ needles, compared with $45^{\circ}$-angle inserted $6 \mathrm{~mm}$ needles (42\% vs $5 \%$ and $24 \%$ vs $5 \%$, $\mathrm{p}<0.001$ ). Even with $4 \mathrm{~mm}$ needles, there is $20.2 \%$ chance of injecting intramuscular when pinched skinfold technique is not applied, and this rate doubles with $5 \mathrm{~mm}$ and triples with $6 \mathrm{~mm}$ needles. The included studies do not report significant changes in the number of adverse events.
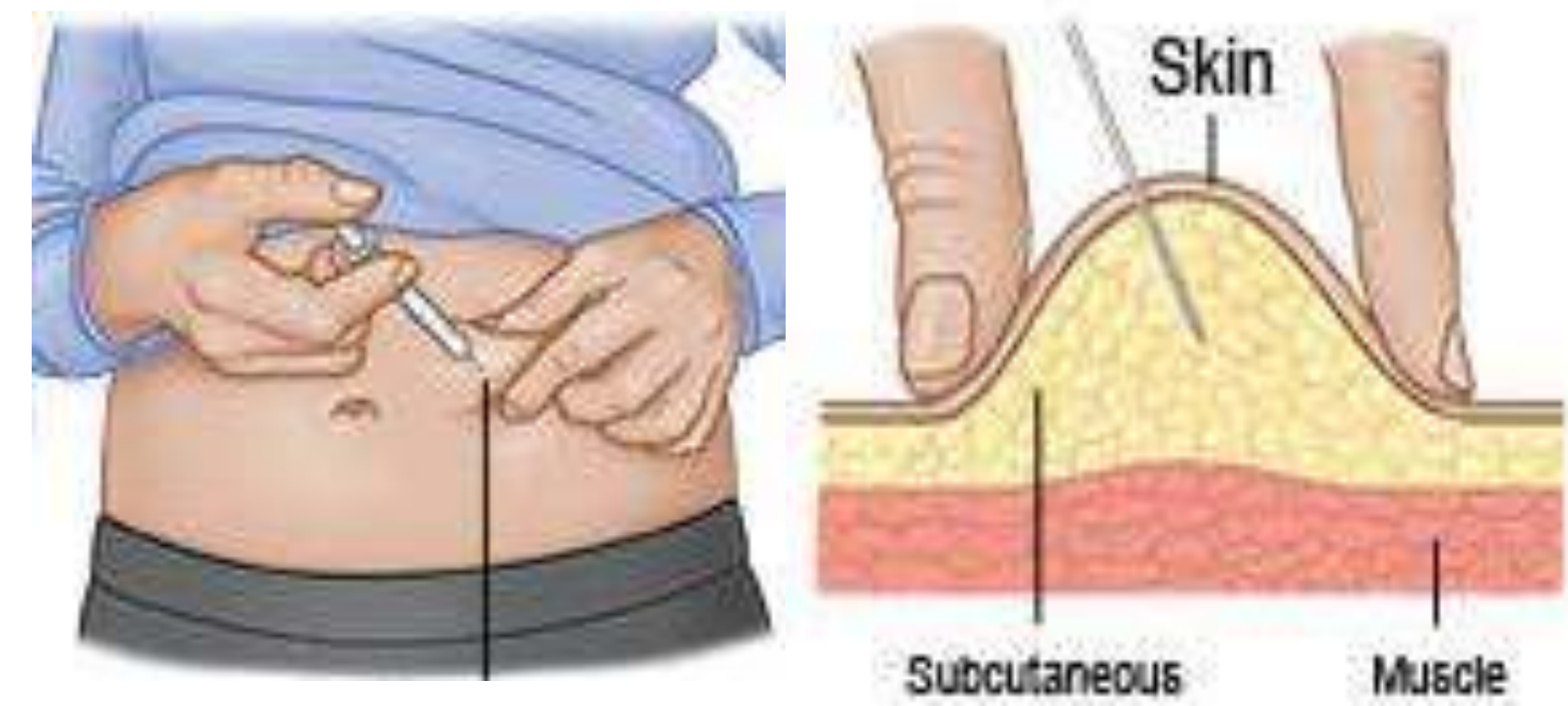

\section{Conclusion}

Based on available evidence we recommend the $45^{\circ}$-angle pinched skinfold technique and $4 \mathrm{~mm}$ needles when providing a subcutaneous injection in pediatric patients. 